\title{
Radiation-Induced Charge Effects in Buried Oxides with Different Processing Treatments*
}

\author{
Christine A. Pennise and H. Edwin Boesch, Jr. \\ U.S. Army Research Laboratory \\ 2800 Powder Mill Road \\ Adelphi, MD 20783-1197 \\ George Goetz and John B. McKitterick \\ AlliedSignal Aerospace Company \\ 9140 Old Annapolis Road \\ Columbia, MD 21045-1998
}

\begin{abstract}
We characterize the radiation-induced charge trapping and transport properties of the buried-oxide (BOX) layer using the photocurrent response technique and capacitance-voltage shift measurements for a variety of silicon-on-insulator (SOI) materials. We observe dramatic differences as a result of different BOX processing conditions. The radiation response of the SOI as a whole is shown to be consistent with the basic properties of the material(s) contained in the BOX layer. In comparison to standard separation by implantation of oxygen (SIMOX) material, SIMOX receiving a supplemental oxygen implant and lowtemperature anneal produces large normalized photocurrent values indicating that both radiation-generated charge carriers move through the BOX. Of the materials examined, the bond-andetch-back silicon-on-insulator (BESOI) material containing a silicon nitride layer produced the lowest normalized photocurrents, heavy trapping of both carriers. By comparison BESOI with thermal oxide layers traps neither carrier in the oxide bulk. The results of this study should be considered in the design of radiation-hardened components and when considering processing variations.
\end{abstract}

\section{INTRODUCTION}

The photoconduction current technique is an important tool in the analysis of the charge-trapping and transport properties in the buried-oxide (BOX) layer of silicon-on-insulator (SOI) material. This method, developed in our earlier work [1], provides a way to determine the amount of radiation-generated charge moving through the BOX layer. The experiment measures the photocurrent, $I_{\mathrm{cm}}$, resulting from the motion of the $\mathrm{x}$-raygenerated electrons and holes in the BOX. In our analysis, $I_{\mathrm{cm}}$ is normalized to a theoretic maximum value, $I_{c o}$, yielding a fraction $I_{c m} / I_{c o}$. Values of the fractional current around 1.0 indicate that both radiation-generated electrons and holes move the maximum distance through the oxide and that very little or no trapping occurs in the oxide bulk. In a bulk oxide where one charge carrier is heavily trapped and the other is highly mobile, we expect a value of $I_{c m} / I_{c o} \approx 0.5$.

\footnotetext{
*This work was supported by the Defense Nuclear Agency.
}

In our previous work [2], we examined bond-and-etch-back silicon-on-insulator (BESOI) material from several different vendors. We recorded a range of values for the normalized currents, depending on the vendor, with $I_{c m} / I_{c o}$ typically in the range 0.7 to 1.1. Using standard pre- and post-irradiation capacitancevoltage $(\mathrm{C}-\mathrm{V})$ measurements, large mid-gap voltage shifts, $\Delta V_{m g}$, were also measured. These results led us to conclude that most of the radiation-generated charge carriers move through the BOX layer, but that a substantial fraction become trapped at one of the interfaces.

Separation by implantation of oxygen (SIMOX) is the leading SOI material for future radiation-hardened microelectronics. Much research has been performed on standard SIMOX material and we, along with many others, have shown that this material traps holes in the bulk oxide layer very heavily and that the electrons move large distances in the BOX [1]. Through the use of the photocurrent technique, measurements obtained on all SIMOX material examined to date yield values of $I_{c m} / I_{c o} \approx$ $0.55 \pm 0.05$, consistent with this interpretation of the transport properties. Additionally, large $\Delta V_{m g}$ relative to dose are measured in this material, indicating a large fraction of hole trapping.

\section{EXPERIMENTAL DETAIS}

\section{A. Materials Examined}

The SOI materials examined in this study include both BESOI and SIMOX. AlliedSignal Aerospace Company (AS) made a series of BESOI materials with a BOX layer where the oxide on either the handle, the seed, or both received special treatment. Standard BESOI, containing a thin layer of thermal oxide on both the handle and the seed wafers, was made at Chalmers University of Technology, Gorteborg, Sweden. The SIMOX material, made at Ibis Technology Corporation, is single-implant SIMOX that has received an additional oxygen implant and anneal. Table 1 gives a complete description of all materials examined.

U.S. Government work not protected by U.S. copyright 
Table 1. Sample Description.

BESOI

AS Material:

(B2) The Control

Seed wafer $-50 \mathrm{~nm}$ thermal oxide grown at $850^{\circ} \mathrm{C}$ in steam with TCA. Handle wafer- $364 \mathrm{~nm}$ thermal oxide grown at $850^{\circ} \mathrm{C}$ in steam with TCA.

(B5) Nitrided Seed Oxide

Seed wafer- $50 \mathrm{~nm}$ thermal oxide grown at $850^{\circ} \mathrm{C}$ in steam with $\mathrm{TCA}$; nitrided in ammonia at $900^{\circ} \mathrm{C}$ for 30 min. Handle wafer-same as B2 handle.

(B7) RNO Seed Oxide

Seed wafer- $50 \mathrm{~nm}$ thermal oxide grown at $850^{\circ} \mathrm{C}$ in steam with TCA; nitrided in ammonia at $900^{\circ} \mathrm{C}$ for 30 min, followed by oxidation at $850^{\circ} \mathrm{C}$ for $10 \mathrm{~min}$. Handle wafer-same as B2 handle.

(B8) Both Oxides Nitrided

Seed wafer-same as B5 seed. Handle wafer- $364 \mathrm{~nm}$ thermal oxide grown at $850^{\circ} \mathrm{C}$ in steam with TCA; nitrided in ammonia at $1100^{\circ} \mathrm{C}$ for $6 \mathrm{hr}$.

(B9) Handle Oxide Nitrided

Seed wafer-same as B2 seed. Handle wafer-same as B8 handle.

(B10) Enhanced Bond

Both seed and handle oxides are the same as B2; both given a proprietary bond strength enhancing treatment.

(B12) Silicon Nitride Seed

Seed wafer $-50 \mathrm{~nm}$ thermal oxide grown at $850^{\circ} \mathrm{C}$ in steam with TCA; $160 \mathrm{~nm}$ of silicon nitride deposited by chemical vapor deposition (CVD). Handle wafer-298.7 nm thermal oxide grown at $850^{\circ} \mathrm{C}$ in steam with TCA.

(B13) Silicon Nitride Handle

Seed wafer-same as B2 seed. Handle wafer-298.7 $\mathrm{nm}$ thermal oxide grown at $850^{\circ} \mathrm{C}$ in steam with TCA; $160 \mathrm{~nm}$ CVD silicon nitride.

(B14) Reoxidized Silicon Nitride Handle

Seed wafer-same as B2 seed. Handle wafer-298.7 nm thermal oxide grown at $850^{\circ} \mathrm{C}$ in steam with TCA; $160 \mathrm{~nm}$ CVD silicon nitride reoxidized at $850^{\circ} \mathrm{C}$ in steam.

Swedish Material:

Both seed and handle wafers contain thermal oxides $\approx 47 \mathrm{~nm}$ thick, grown at $900^{\circ} \mathrm{C}$ in dry oxygen, followed by an anneal at $900^{\circ} \mathrm{C}$ for $45 \mathrm{~min}$ in nitrogen gas.

SIMOX

Ibis Material:

Standard $1.8 \times 10^{18} \mathrm{~cm}^{-2}$ single oxygen implant followed by a supplemental $1.0 \times 10^{17} \mathrm{~cm}^{-2}$ implant and a $1 \mathrm{hr} 1000^{\circ} \mathrm{C}$ anneal; BOX thickness $403.4 \mathrm{~nm}$.

AS fabricated devices on their BESOI, and the Army Research Laboratory (ARL) performed the die attach and wire bonding; capacitors with active areas of 0.003 and $0.01 \mathrm{~cm}^{2}$ were examined in this study. Sample preparation was performed at ARL on the Swedish BESOI and the Ibis SIMOX materials; capacitors with areas of 0.00209 and $0.00581 \mathrm{~cm}^{2}$ were fabricated.

\section{B. Experimental Techniques}

We used the photoconduction current technique and performed C-V measurements as a function of dose to study the radiation-induced charge trapping and transport in this series of SOI materials. The photocurrent experiments were performed 
using the ARACOR 10-keV $\mathrm{x}$-ray machine as the radiation source, at room temperature unless noted otherwise. Several samples from each material were examined, and with the exception of the Swedish BESOI, each sample produced essentially the same $I_{c m} / I_{c o}$. Therefore, where appropriate, results obtained from one capacitor will be shown in the figure to illustrate characteristic behavior obtained from that material.

Room-temperature C-V measurements were taken on a representative set of AS BESOI samples and the Ibis SIMOX material using the ARACOR as the radiation source. Fast C-V measurements were also performed on the AS BESOI wafers that contained the $\mathrm{Si}_{3} \mathrm{~N}_{4}$ and the reoxidized $\mathrm{Si}_{3} \mathrm{~N}_{4}$ as the insulating layer, wafers B12, B13, and B14. These measurements were taken as a function of time and temperature after exposure to a LINAC radiation pulse [3].

\section{RESULTS AND DISCUSSION}

\section{A. Photoconduction Current Measurements}

\section{AlliedSignal BESOI}

Figures 1, 2, and 3 show results from the photoconduction current measurements performed on the AS material. Normalized photocurrents obtained from the control samples range from about 0.86 at fields less than $1 \mathrm{MV} / \mathrm{cm}$, to slightly above 1.0 at the higher fields. (These results essentially repeat measurements previously reported [2] on similar AS BESOI material.) $I_{c m} / I_{c o}$ for the control material is shown in figures 1 and 3 , and can be used as a standard for comparison.
Figure 1 illustrates the effect nitridation of the handle and/or seed oxide has on the normalized photocurrents; the figure shows data from samples with either the handle, seed, or both oxides nitrided. Notice that the nitridation of the oxide(s) reduces $I_{\mathrm{cm}}$ $I_{c o}$ considerably. The greatest effect on $I_{c m} / I_{c o}$ seems to have occurred in the case of the nitrided handle oxide (B9) at the lower fields; overall $I_{c m} / I_{c o}$ ranges from about 0.45 to 0.7 for this sample. Results obtained on samples with the nitrided seed (B5) and samples with both oxides nitrided (B8) are essentially the same, with values of $I_{c m} / I_{c o}$ in the range of 0.55 to 0.7 . The lower fractional currents observed in these oxides are consistent with the fact that the nitridation process incorporates nitrogen and hydrogen into the oxide and results in the creation of electron traps; see Chaiyasena et al. [4], for example.

The presence of a stoichiometric $\mathrm{Si}_{3} \mathrm{~N}_{4}$ layer in the BOX has a dramatic effect on the normalized photocurrents, as illustrated in figure 2 , where $I_{c m} / I_{c o}$ ranges from roughly 0.3 to 0.4 . Of these three wafers, the sample with the silicon nitride layer on the handle oxide, B13, yielded the highest photocurrents with values of $I_{c m} / I_{c o}$ roughly 0.4 for all but the lowest fields. The wafer with the $\mathrm{Si}_{3} \mathrm{~N}_{4}$ layer on the seed, B12, yielded the lowest photocurrents with values of $I_{c m} / I_{c o}$ about 0.3 for all but the lowest fields. The wafer receiving the reoxidation of the silicon nitrided layer, B14, produced intermediate values of $I_{\mathrm{cm}} / I_{c o}$. We anticipated that sample B14 would show the highest photocurrents of the three, since the reoxidation process is known to diminish the presence of traps in nitrided oxides [4]. As shown, the reoxidation of the $\mathrm{Si}_{3} \mathrm{~N}_{4}$ had litule if any effect on the trapping properties of the insulating layer in these materials. This may be due to differences in the trapping species or mechanisms

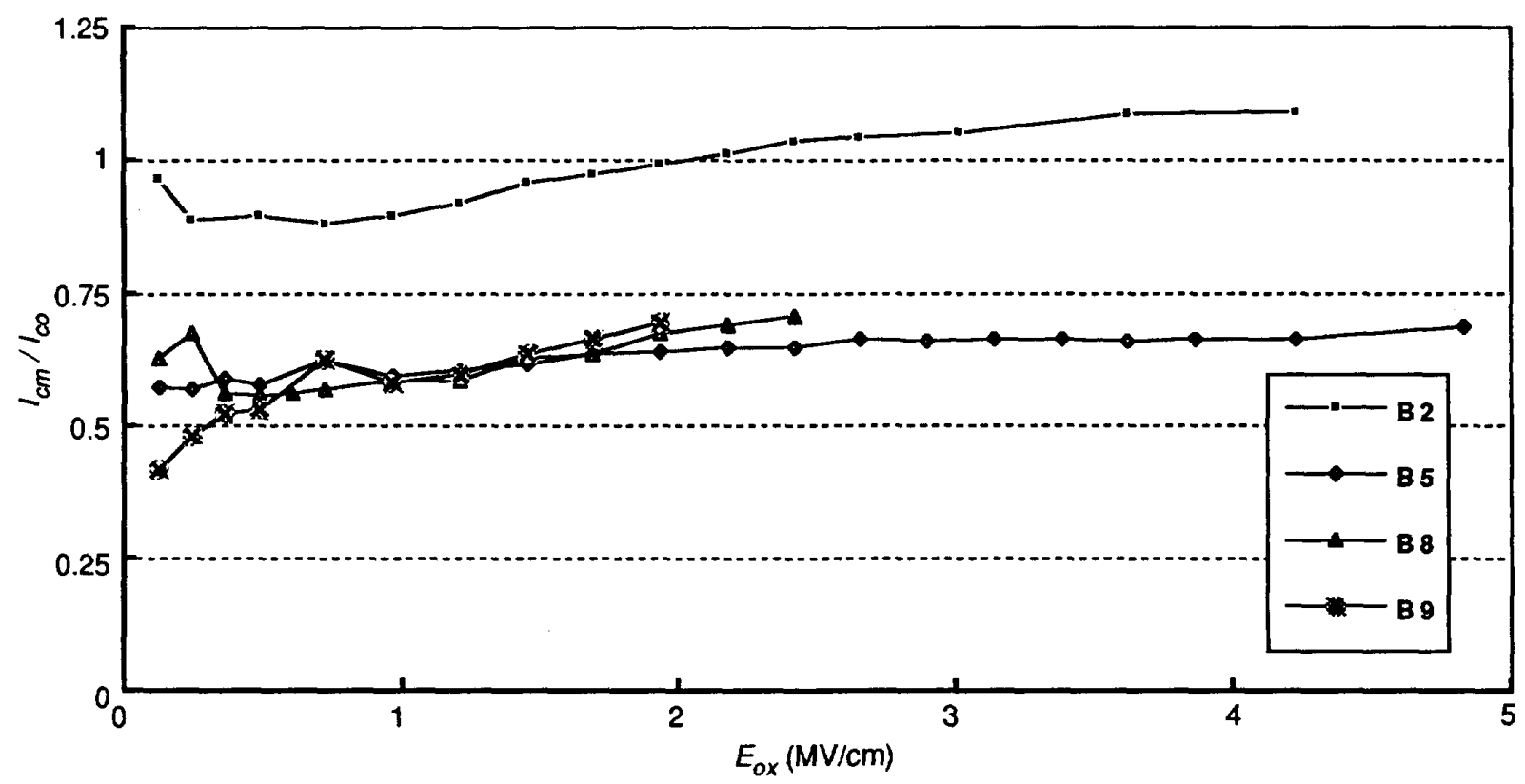

Figure 1. Normalized photoconduction currents, $I_{c m} / I_{c o}$, as a function of field, $E_{o x}$ for the AS material: BOX with seed (B5), handle (B9), or both oxides nitrided (B8). 


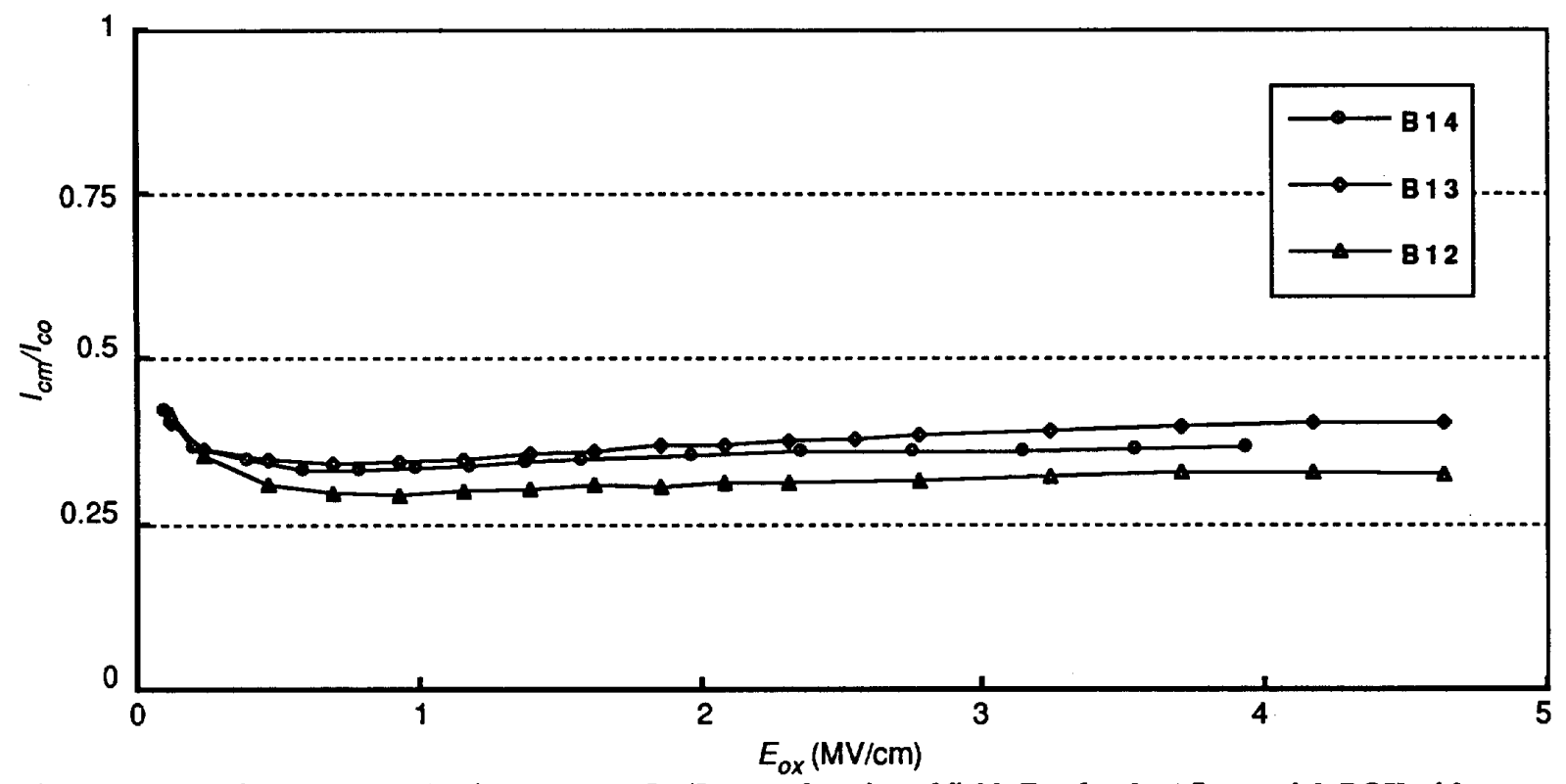

Figure 2. Normalized photoconduction currents, $I_{c m} / I_{c o}$, as a function of field, $E_{o x}$ for the AS material: BOX with a $\mathrm{Si}_{3} \mathrm{~N}_{4}$ layer on seed wafer (B12), on handle wafer (B13) and reoxidized $\mathrm{Si}_{3} \mathrm{~N}_{4}$ on handle wafer (B14).

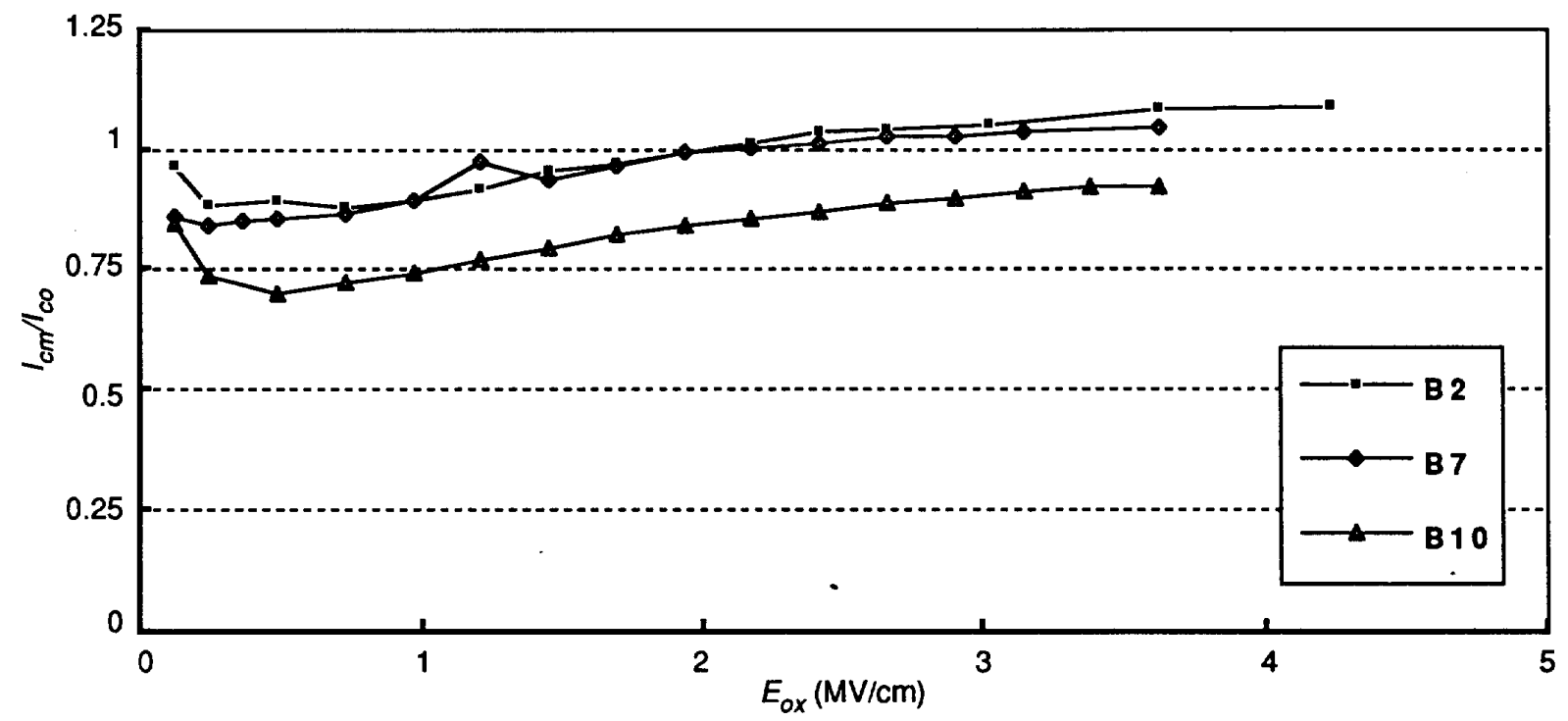

Figure 3. Normalized photoconduction currents, $I_{c m} / I_{c o}$, as a function of field, $E_{o x}$ for the AS material: BOX with RNO (B7) and enhanced-bond (B10) oxides.

in this material or possibly an incomplete reoxidation process. Overall the rather low values of $I_{c m} / I_{c o}$ were anticipated, since $\mathrm{Si}_{3} \mathrm{~N}_{4}$ is known to heavily trap both holes and electrons [5], resulting in a greatly reduced photocurrent to flow in the insulating layer.

Figure 3 shows the results of the photocurrent experiment on the remaining AS materials: the reoxidized nitrided oxide (RNO) wafer (B7) and the enhanced bond wafer (B10). The RNO material produced photocurrents similar to the control material with $I_{c m} / I_{c o}$ ranging from 0.8 to 1.0 . These results are consistent with observations that the reoxidation of nitrided oxides diminishes the density of electron traps created in the nitridation process [4]. Wafer B10 had a BOX layer where the oxides on both the handle and seed wafers have been given a bond strength enhancing treatment and the effects of this treatment on $I_{c m} / I_{c o}$ are also shown in this figure. This procedure reduces the resulting photocurrents, although not as pronouncedly as that of the nitridation of the oxides or the BESOI with the $\mathrm{Si}_{3} \mathrm{~N}_{4}$ layers, with $I_{c m} / I_{c o}$ ranging from 0.65 to 0.95 . Both electron trapping and enhanced hole trapping are known to occur in oxides that have been implanted or doped with impurity ions [6]. From the reduced $I_{c m} / I_{c o}$ measured on B 10, as illustrated in 
figure 3, we can conclude that bond strength enhancing treatment is incorporating some type of impurity ions into the BOX.

To summarize the results obtained on the AS BESOI materials, we note the strong dependence of the radiation response of the BESOI on the treatment received by the handle, seed, or both oxides in the BOX. Normalized photocurrents measured on the control sample (B2) were around 1.0, which is consistent with previous measurements and with what is expected for thermally grown oxides. The most dramatic effect was observed for the BESOI structures containing the $\mathrm{Si}_{3} \mathrm{~N}_{4}$ in the BOX layer, where values of $I_{c m} / I_{c o}$ on the order of 0.35 were recorded. Nitridation of the handle, seed, or both oxides was also shown to cause a rather large decrease in $I_{c m} / I_{c o}$, over that of the control, with values ranging from about 0.55 to 0.7 . These decreased photocurrents, for the BESOI containing the $\mathrm{Si}_{3} \mathrm{~N}_{4}$ or the nitrided oxides, were somewhat anticipated due to the known trapping characteristics of the material contained in the BOX. In a similarly consistent fashion, the BESOI material with RNO BOX produced large photocurrents, with $I_{c m} / I_{c o} \approx 0.8-1.1$, which is essentially equal to that of the control. We have observed in each case that the radiation-induced characteristics of the overall structure are consistent with the known behavior (properties in the bulk and at the interfaces) of the material contained in the BOX layer.

\section{Swedish BESOI}

We also examined BESOI material obtained from a group at Chalmers University. The BOX in this material contains thermally grown oxide layers of equal thickness on the handle and seed wafers, and therefore the bond is located at the center of the BOX layer. This material has exhibited strong charging effects at or close to the bond when the dielectric is injected with charge by Fowler-Nordheim tunneling [7]. The strong trap- ping in the vicinity of the bond and its location at the center of the BOX has caused greatly reduced $I_{c m} / I_{c o}$, in comparison to similar BESOI materials, as illustrated in figure 4. We observed more sample-to-sample variation in this material than normally recorded, therefore results from two capacitors on the Swedish BESOI material are plotted with the AS control material. Recall the photoconduction current technique is a method to measure the charge moving through the oxide; therefore if the bond region is acting as a heavy trapping site, a large percentage of the carriers will become trapped there, resulting in a reduced $I_{c m}$.

\section{SIMOX}

The Ibis SIMOX material we investigated received a supplemental oxygen implant followed by a low-temperature anneal, as detailed in table 1 . This additional implant causes a dramatic change in the charge-trapping and transport characteristics of the SIMOX BOX. As shown in figure 5, the fractional photocurrents measured in this material are greatly increased over standard SIMOX, with $I_{c m} / I_{c o} \approx 0.85-1.0$. The results of the photocurrent experiment performed on the supplemental oxygen SIMOX material at low temperature $(T \approx 155 \mathrm{~K})$ are also shown in this figure. For measurement times of the experiment, this temperature should immobilize most of the holes and one would expect to measure $I_{c m} / I_{c o} \approx 0.5$. As illustrated, we measure roughly 0.55 for most bias conditions; the increase at higher fields is likely due to either the sample warming up over the duration of the experiment or possibly the accelerated recovery rate of trapped holes with increasing field [6]. By comparing the low-temperature photocurrents with those at high temperature $-I_{c m} / I_{c o}$ is about 0.55 and 0.9 , respectively-we see clearly that at room temperature the holes are moving through the BOX in the supplemental SIMOX.

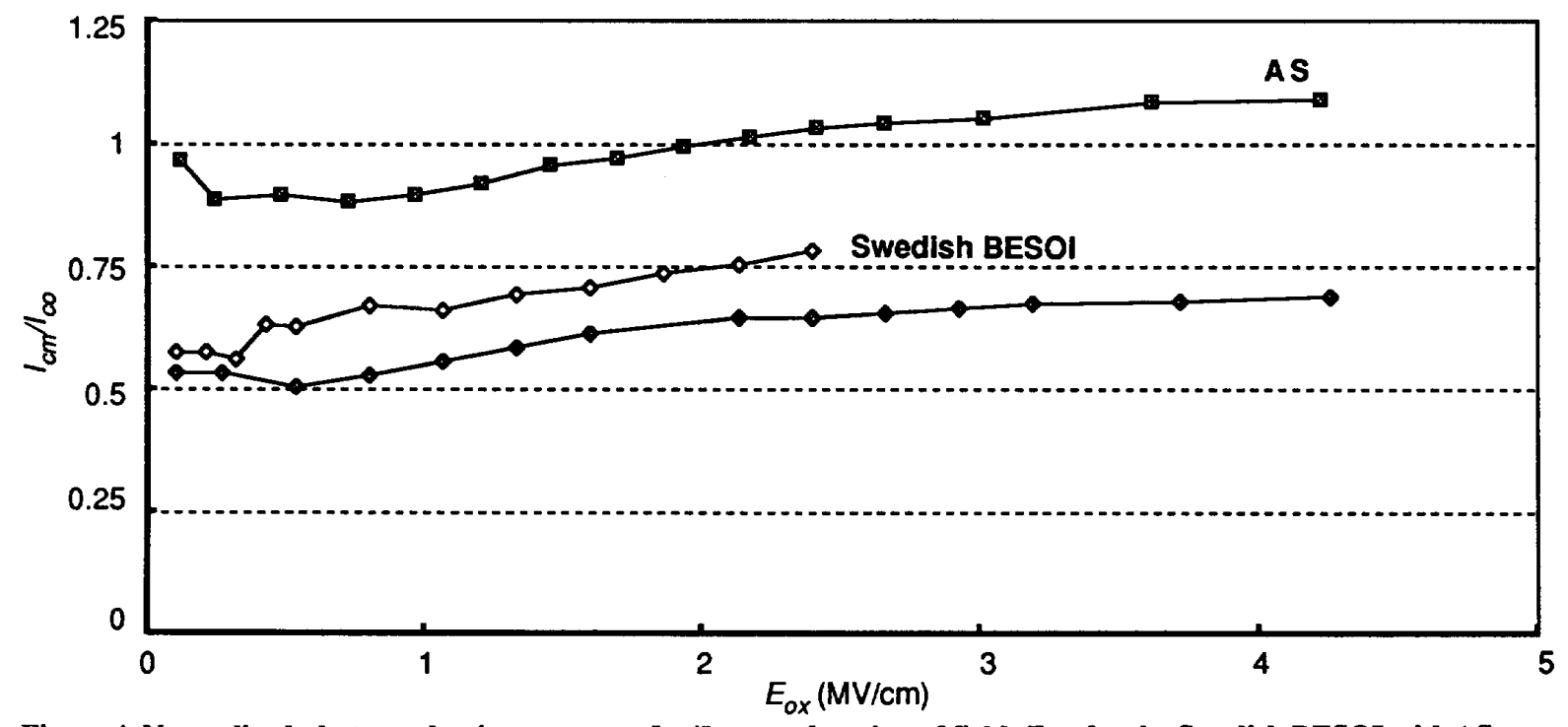

Figure 4. Normalized photoconduction currents, $I_{c m} / I_{c o s}$ as a function of field, $E_{o x}$, for the Swedish BESOI with AS control shown for reference. 


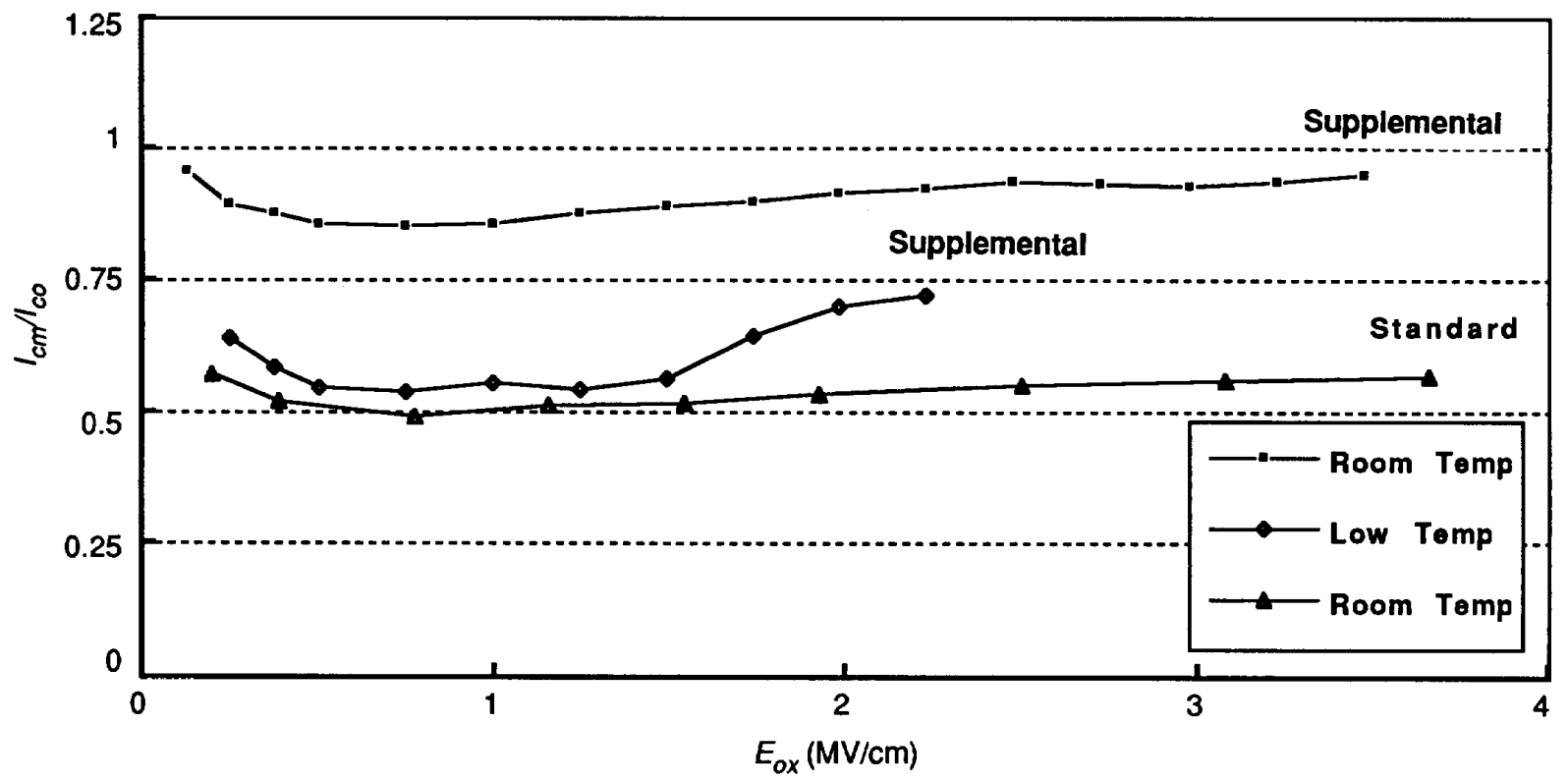

Figure 5. Normalized photoconduction currents, $I_{c m} / I_{c o}$, at room and low temperature, as a function of field, $E_{o x}$ for Ibis supplemental and standard SIMOX material.

\section{B. C-V Measurements and Modeling}

With the $10-\mathrm{keV} \mathrm{x}$-ray machine as the radiation source, we performed pre- and post-irradiation C-V measurements on capacitors from a representative set of the AS BESOI material including: the control, B2; RNO on the seed oxide, B7; both handle and seed oxides nitrided, $\mathrm{B} 8 ; \mathrm{Si}_{3} \mathrm{~N}_{4}$ on seed oxide, $\mathrm{B} 12$; and the Ibis SIMOX. The light doping of the top Si layer in the AS BESOI material allows us to monitor the voltage shifts at both the bottom and the top interfaces. The top Si layer of the Ibis material was heavily doped and therefore $\Delta V_{m g}$ could be determined at the back interface only. We irradiated samples at room temperature to $5 \mathrm{krad}\left(\mathrm{SiO}_{2}\right)$ under an applied field of +1.0 or $-1 \mathrm{MV} / \mathrm{cm}$ and measured $\Delta V_{m g}$ for each case, as shown in table 2. We kept the dose low and the applied field high to avoid space-charge build-up and to insure a linear voltage shift with dose. The voltage shift at the bottom interface under positive and negative bias is designated $\Delta V_{b}{ }^{+}$and $\Delta V_{b}{ }^{-}$, respectively; similarly, the top interface is designated $\Delta V_{t}^{+}$and $\Delta V_{t}^{-}$.

Due to the vast differences in the properties of the materials examined, we use two different models to characterize the charge trapping and transport in these materials. One, the model developed by Boesch and Taylor [3], we used to extract the electron and hole mean-free path to capture by traps in the bulk oxide, $S_{e}$ and $S_{h}$, respectively, and the fraction of x-ray generated electrons and holes that reach the bottom interface, $f_{b e}$ and $f_{b h}$, or the top interface, $f_{l e}$ and $f_{t h}$, and are trapped there. The modeled bulk oxide and interface trapping parameters, shown in table 3 , represent the best fit to the measured data. We developed a second model to characterize the three-layer oxide/silicon nitride/ oxide BESOI structures; it accounts for the large amount of trapping present in this material. This model assumes a three-layer structure with no hole motion in the oxide layers, no electron or hole motion in the nitride layer, and 100-percent electron trapping at the oxide/nitride interfaces.

\section{AlliedSignal BESOI}

A comparison of tables 2 and 3 demonstrates that the model produces a reasonable fit to the data for wafers B2, B7, and B8-showing the trend to more trapping, as expected-from the control to the RNO and nitrided BOX materials. For wafer $\mathrm{B} 12$, the modeled trapping parameters indicate heavy trapping in the insulating layer (very low values for both $S_{e}$ and $S_{h}$ ), as anticipated. However, by comparing the measured and predicted values of $\Delta V_{m g}$, as shown in tables 2 and 3, respectively, we see this model-which assumes a single oxide layer-is inadequate to describe this case as indicated by the poor fit to the measured data. This is because the $\mathrm{Si}_{3} \mathrm{~N}_{4}$ layer behaves substantially different from a thermal oxide and must be treated as a second layer in the BOX.

We obtain additional information about the charge-trapping properties of the AS material with the $\mathrm{Si}_{3} \mathrm{~N}_{4}$ in the BOX layer from our analysis of the fast $\mathrm{C}-\mathrm{V}$ data. Figure 6 shows $\Delta V_{m}$ plotted as a function of time after exposure to 4- $\mu$ s LINAC radiation pulses for sample $\mathrm{B} 13\left(\mathrm{Si}_{3} \mathrm{~N}_{4}\right.$ on the handle wafer), irradiated at various temperatures under +1 or $-1 \mathrm{MV} / \mathrm{cm}$ applied field. The figure shows $\Delta V_{m g}(t)$ for (a) the bottom interface and (b) the top interface. We obtained equivalent results for AS wafers B12 $\left(\mathrm{Si}_{3} \mathrm{~N}_{4}\right.$ on seed) and B14 (reoxidized $\mathrm{Si}_{3} \mathrm{~N}_{4}$ on handle). Compared with similar measurements on other BESOI materials [3], $\Delta V_{m g}$ shows very little change as a function of time and temperature. At low temperatures (near $120 \mathrm{~K}$ ) and at early times $(0.2 \mathrm{~ms})$ after the radiation pulse, the radiation-generated holes 
are essentially immobile and have not had time to move from their points of generation in the insulator bulk, whereas at $300 \mathrm{~K}$ and late times ( $400 \mathrm{~s})$, the holes should have completed any motion through the insulator [6]. Measured values of $\Delta V_{m g}$ at $0.2 \mathrm{~ms}$ near $120 \mathrm{~K}$ and at $400 \mathrm{~s}$ and $300 \mathrm{~K}$ are listed in table 4 for AS samples B12, B13, and B14. As noted previously for the AS B 12 material irradiated with $\mathrm{X}$-rays (see table 3), these C-V shifts cannot be satisfactorily fit using the single-layer trapping model. Using the model that assumes a three-layer structure, voltage shifts for devices on these wafers have been calculated to be $\Delta V_{b}{ }^{+}=-10.1, \Delta V_{b}{ }^{-}=-18.3, \Delta V_{t}^{+}=5.4$, and $\Delta V_{t}^{-}=-10.1 \mathrm{~V}$, in reasonable agreement with the measured values for both the early/low and late/high time and temperature conditions. In addition, the predicted $I_{c m} / I_{c o}$ from this model for the oxide/ nitride/oxide structure is about 0.3 at $1 \mathrm{MV} / \mathrm{cm}$, which is in good agreement with the results shown in figure 2 . These results support a model for the nitride and reoxidized nitride BESOI structures in which the nitride or reoxidized nitride layer traps both carriers efficiently. Curiously, the addition of the nitride layer also seems to result in substantial bulk hole trapping in the oxide layers.

Table 2. Measured voltage shifts and normalized currents of x-ray irradiated samples.

\begin{tabular}{lcclll}
\hline Sample & $\Delta V_{b}^{+}(\mathrm{V})$ & $\Delta V_{b}^{-}(\mathrm{V})$ & $\Delta V_{t}^{+}(\mathrm{V})$ & $\Delta V_{t}^{-}(\mathrm{V})$ & $I_{c m^{\prime} I_{c o}}$ \\
\hline AS B2 & -3.0 & -4.0 & +1.25 & -10.0 & 0.9 \\
AS B7 & -6.0 & -5.5 & -5.5 & -15.7 & 0.9 \\
AS B8 & -8.75 & -8.0 & -4.5 & -10.0 & 0.6 \\
AS B12 & -5.0 & -7.75 & +1.5 & -1.0 & 0.3 \\
Ibis & -12.75 & +2.5 & - & - & 0.85 \\
\hline
\end{tabular}

Table 3. Modeled trapping parameters and predicted values of voltage shifts and normalized photocurrents.

\begin{tabular}{lcclllllllll}
\hline Sample & $S_{h}(\mathrm{~nm})$ & $S_{e}(\mathrm{~nm})$ & $f_{b h}$ & $f_{b e}$ & $f_{t h}$ & $f_{t e}$ & $\Delta V_{b}+(\mathrm{V})$ & $\Delta V_{b}-(\mathrm{V})$ & $\Delta V_{t}^{+}(\mathrm{V})$ & $\Delta V_{t}^{-}(\mathrm{V})$ & $I_{c m} / I_{c o}$ \\
\hline AS B2 & 500 & 30,000 & 0 & 0 & 0.7 & 0.19 & -3.09 & -1.65 & +1.2 & -10.3 & 0.83 \\
AS B7 & 137.5 & 20,000 & 0 & 0 & 1.0 & 0 & -6.2 & -4.0 & -4.0 & -11.0 & 0.7 \\
AS B8 & 60 & 20,000 & 0.7 & 0 & 1.0 & 0.075 & -8.74 & -5.59 & -4.47 & -9.4 & 0.612 \\
AS B12 & 0.1 & 270 & 0 & 0 & 0 & 0.55 & -5.17 & -3.06 & +1.47 & -5.17 & 0.27 \\
Ibis & 10,000 & 10,000 & 0.9 & 0.168 & - & - & -12.78 & +2.46 & - & - & 0.97 \\
\hline
\end{tabular}

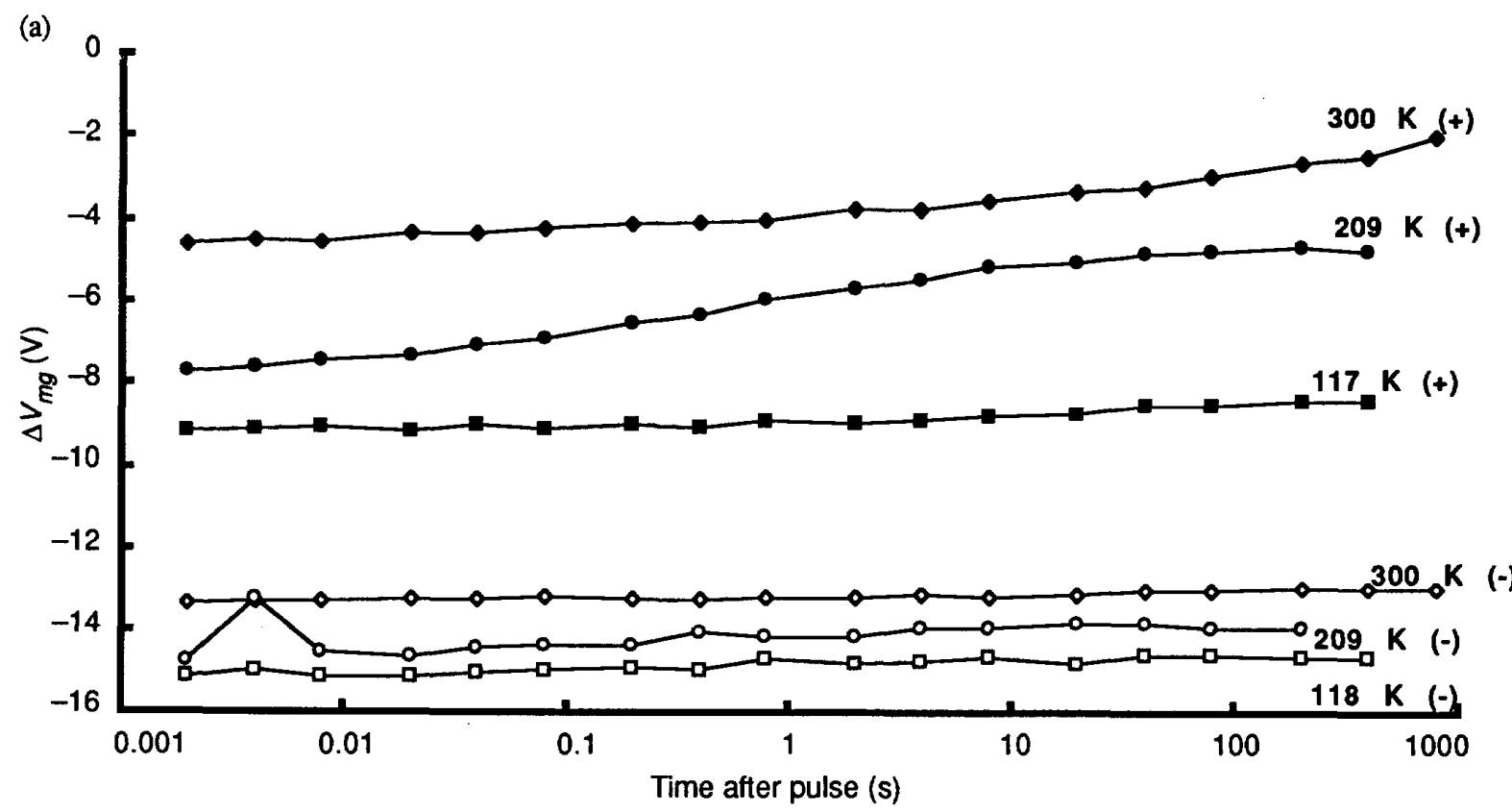

Figure 6. Results from LINAC fast C-V measurements for AS BESOI B13: $\Delta V_{m g}$ as a function of time and temperature for (a) bottom interface. 


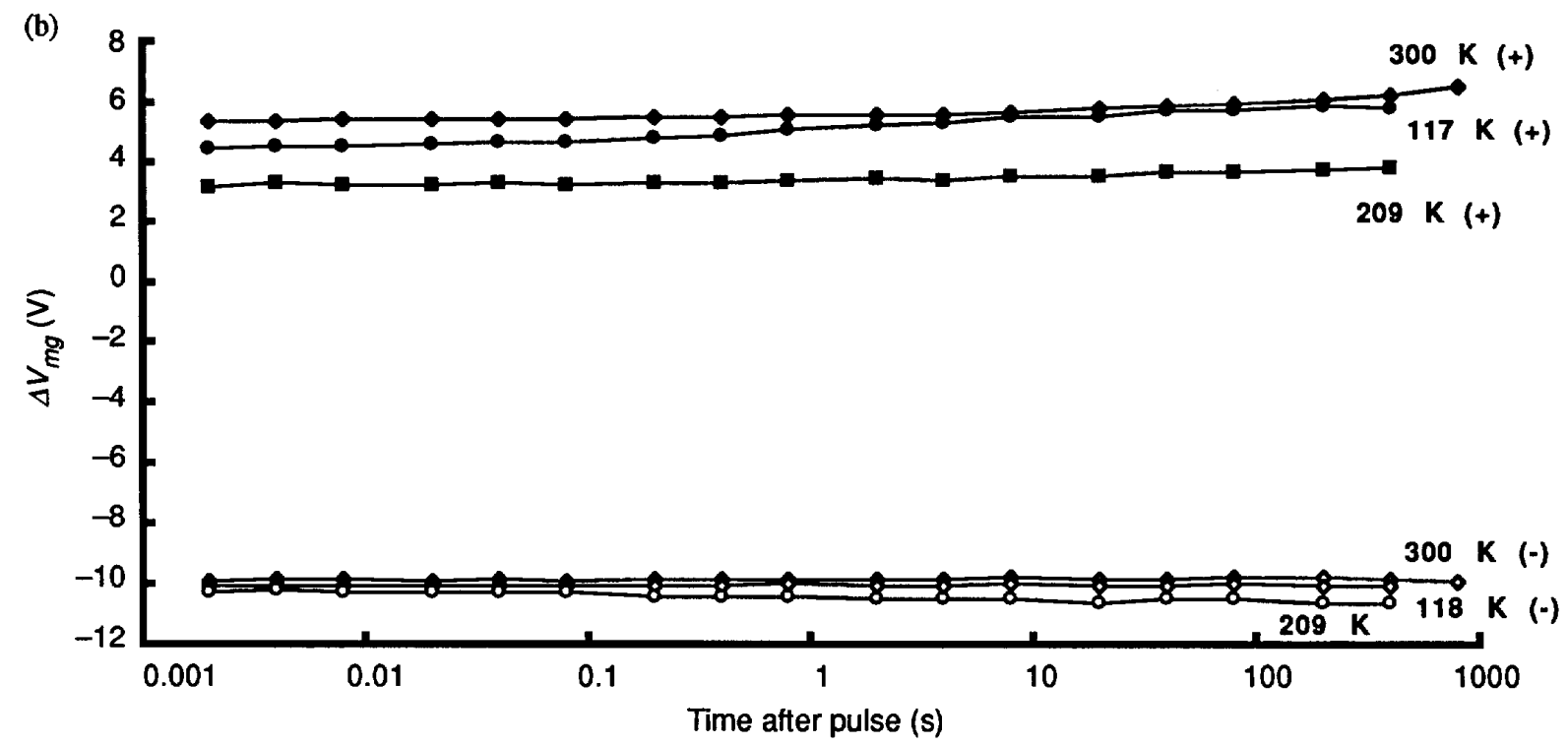

Figure 6. Results from LINAC fast C-V measurements for AS BESOI B13: $\Delta V_{m g}$ as a function of time and temperature (b) top interface.

Table 4. Measured voltage shifts for LINAC irradiated samples.

(Note: (a) refers to measurements taken $02 \mathrm{~ms}$ after LINAC pulse at $117 \mathrm{~K}$ and (b) refers to measurements taken 400 s afier LINAC pulse at $300 \mathrm{~K}$.)

\begin{tabular}{lcccr}
\hline Sample & $\Delta V_{b}{ }^{+}(\mathrm{V})$ & $\Delta V_{b}{ }^{-}(\mathrm{V})$ & $\Delta V_{t}^{+}(\mathrm{V})$ & $\Delta V_{t}^{-}(\mathrm{V})$ \\
\hline (a) AS B12 & -8.9 & -16.7 & +4.0 & -9.5 \\
(b) & -9.0 & -12.9 & +4.3 & -6.0 \\
(a) AS B13 & -9.2 & -15.3 & +3.2 & -10.1 \\
(b) & -2.4 & -12.9 & +6.2 & -9.8 \\
(a) AS B14 & -8.7 & -17.4 & +3.6 & -10.5 \\
(b) & -1.0 & -13.2 & +7.7 & -10.3 \\
\hline
\end{tabular}

\section{SIMOX}

The room- and low-temperature photocurrent results clearly indicate that the Ibis SIMOX material is trapping little, if any, $\mathrm{x}$-ray-generated electrons or holes in the BOX layer. Additionally, by modeling conventional room temperature $\mathrm{C}-\mathrm{V}$ measurements, using the single layer, bulk oxide, and interfacial trapping model, we further verify these results. From the values of the model parameters that yield the best fit to the measured midgap voltage shifts, we conclude that both the electrons and holes move through the oxide indicated by the large values of both $S_{e}$ and $S_{h}$ (see table 3). This material does show evidence of trapping at the interface, indicated by the large voltage shifts relative to absorbed dose and the modeled values of the fraction of charge trapped at the interface. These results lead us to conclude that the additional oxygen implant is successful in greatly reducing the number of hole traps present in the bulk oxide. The mechanism responsible for reducing the number of traps may be the additional oxygen supplied by the supplemental implant filling oxygen vacancies that were created by damage during the initial SIMOX process. This process may be further aided by the additional lower temperature anneal $\left(T=1000^{\circ} \mathrm{C}\right.$, an optimum temperature for growth of thermal $\mathrm{SiO}_{2}$ ).

\section{ConClusions}

We examined the radiation-induced charge trapping and transport characteristics of a variety of SOI materials, using the photoconduction current technique and analysis of $\Delta V_{m g}$ obtained from the total dose $\mathrm{C}-\mathrm{V}$ measurements. We have shown the radiation response of the material to be very dependent on the treatment that the BOX oxides have received, and to be consistent with the basic properties of the individual layers and their interfaces. We have also shown that techniques successful in reducing the number of traps present in individual thermal oxide layers, such as the RNO process, are also successful when applied to a complete BESOI structure. We observed that processing and special treatment of the BOX can significantly affect $I_{\mathrm{cm}}$ ' $I_{c o}$ and the overall radiation response of the material.

In this study we examined a series of nine AS BESOI wafers, each containing a different BOX layer. The measured photocurrents vary considerably, with values as high as $I_{c m} / I_{c o} \approx 1.0$ for the control and the wafer receiving the RNO treatment, to values as low as $I_{c m} / I_{c o} \approx 0.35 \pm 0.05$ for the three wafers with the $\mathrm{Si}_{3} \mathrm{~N}_{4}$ in the BOX. The set of three wafers where either the handle, seed, or both oxides were nitrided produced intermediate results, as did the wafer that received the bond strength enhancing treatment. We took C-V measurements on a representative subset of samples and modeled the measured $\Delta V_{m g}$. Both measured and modeled results indicate the least amount of trapping in the bulk and at the interfaces is present in the control material, and the most is in the $\mathrm{Si}_{3} \mathrm{~N}_{4}$ oxide materials, with the other materials showing intermediate amounts of trapping. The trapping characteristics of the BESOI structures were all con- 
sistent with the known properties of the material contained in the BOX, as well as the results from the photocurrent experiment.

Normalized photocurrents obtained from the Swedish BESOI were low in comparison to similar AS material (B2), with values of $I_{c m} / I_{c o}$ ranging from about 0.55 to 0.75 . We attribute the low values of $I_{c m} / I_{c o}$ (and therefore trapping in the bulk oxide) to the observed charging effects at or near the bond [7] and to the location of bond. The strong charging effects in the vicinity of the bond indicate it is acting as a trapping site and its location, at the center of the BOX, is responsible for the low photocurrents. These results indicate the effects that the oxide-oxide bond interface and its location in the BOX can have on the charge-trapping properties of a material and is consistent with conclusions drawn by Boesch et al. [3] in their research on an earlier set of AS BESOI material. Although we are uncertain as to the exact cause of the (heavy) trapping behavior at the bond in the Swedish BESOI, the results from this material clearly indicate the role the bond characteristics play in the radiation response of the material as a whole. The importance of this is evident - the quality and location of the bond must be considered and controlled throughout the BESOI fabrication process.

A striking effect was seen in the case of the Ibis SIMOX exposed to an additional $\mathrm{O}_{2}$ implant and (low-temperature) anneal. We recorded values of $I_{c m} / I_{c o} \approx 0.95 \pm 0.05$ as compared to standard SIMOX examined in the past where $I_{c m} / I_{c o} \approx$ 0.55 [1]. These results indicate there is very little radiationgenerated charge trapping in the bulk BOX layer in the SIMOX receiving the supplemental implant and anneal. This implies both the radiation-generated electrons and the holes are moving very large distances through the BOX. However, the large measured voltage shifts, relative to dose, indicate trapping at the bottom interface of one or both charge carriers. The trapping parameters modeled to best fit the measured $\Delta V_{m g}$ verify the experimental results and reveal a large percentage of the holes and some electrons-about 90 and 17 percent, respectively-are trapped at the interface in this SIMOX.

The supplemental SIMOX is clearly behaving differently than SIMOX produced using the standard procedures. In fact, our results show the charge-trapping and transport characteristics of its BOX to be similar to a soft, thick thermal oxide. The supplemental implant and anneal is the first step in improving the hardness of SIMOX material; these results indicate that processing techniques and conditions that are presently designed to radiation-harden thermally grown oxides may be applied to the supplemental SIMOX with a good chance for success. This is critical if SIMOX is to be the material of choice for radiation-hardened microelectronic components.

The strong connection between the properties and characteristics of the material(s) contained in the $\mathrm{BOX}$ with the over- all radiation response of the SOI structure as a whole is evident from this research. These are important results that should be considered in the design of radiation-hardened components and when planning various processing techniques or variants.

\section{ACKNOWLEDGMENTS}

The authors would like to thank W. Krull of Ibis Technology Corporation for providing us their material; S. Bengtsson and O. Engström of Chalmers University of Technology for their material and useful discussions, and special thanks to B. Dobranski and J. McCullen at our microelectronics facility for preparing the samples.

\section{REFERENCES}

(1) C. A. Pennise and H. E. Boesch, Jr., "Determination of the Charge-Trapping Characteristics of Buried Oxides Using a 10-keV X-Ray Source," IEEE Trans. Nucl. Sci. NS-37, 1990-1994 (December 1990).

(2) C.A. Pennise and H. E. Boesch, Jr., "Photoconduction Measurements of the Charge Trapping and Transport in Bond-and-Etch-Back Buried Oxides," IEEE Trans. Nucl. Sci. NS-39, 2139-2145 (December 1992).

(3) H. E. Boesch, Jr., and T. L. Taylor, "Time-Dependent Radiation-Induced Charge Effects in Wafer-Bonded SOI Buried Oxides," IEEE Trans. Nucl. Sci. NS-39, 2103-2113 (December 1992).

(4) I. A. Chaiyasena, P. M. Lenahan, and G. J. Dunn, "Identification of a Paramagnetic Nitrogen Dangling Bond Defect in Nitrided Silicon Dioxide Films on Silicon," Appl. Phys. Lett. 58 (19), 21412143 (May 1991).

(5) N. Saks, J. M. Killiany, P. R. Reid, and W. D. Baker, "A Radiation Hard MNOS CCD For Low Temperature Applications," IEEE Trans. Nucl. Sci. NS-26, 5074 (December 1979).

(6) F. B. McLean, H. E. Boesch, Jr., and T. R. Oldham, Chapter 3 of Ionizing Radiation Effects in MOS Devices and Circuits, T. P. Ma and P. V. Dressendorfer, eds., Wiley: New York (1989).

(7) A. Jauhiainen, S. Bengtsson, and O. Engström, "Charge Trapping in Wafer Bonded MOS Structures," presented at 22nd European Solid-State Device Research Conference, published in Microelectronic Engineering 19, 597-600 (September 1992). 